\title{
Predictors of low cervical cancer screening among immigrant women in Ontario, Canada
}

\author{
Aisha K Lofters ${ }^{1,2,3,4^{*}}$, Rahim Moineddin ${ }^{1,5,6}$, Stephen W Hwang ${ }^{3,7}$ and Richard H Glazier ${ }^{1,2,3,5,6}$
}

\begin{abstract}
Background: Disparities in cervical cancer screening are known to exist in Ontario, Canada for foreign-born women. The relative importance of various barriers to screening may vary across ethnic groups. This study aimed to determine how predictors of low cervical cancer screening, reflective of sociodemographics, the health care system, and migration, varied by region of origin for Ontario's immigrant women.

Methods: Using a validated billing code algorithm, we determined the proportion of women who were not screened during the three-year period of 2006-2008 among 455864 identified immigrant women living in Ontario's urban centres. We created eight identical multivariate Poisson models, stratified by eight regions of origin for immigrant women. In these models, we adjusted for various sociodemographic, health care-related and migration-related variables. We then used the resulting adjusted relative risks to calculate population-attributable fractions for each variable by region of origin.

Results: Region of origin was not a significant source of effect modification for lack of recent cervical cancer screening. Certain variables were significantly associated with lack of screening across all or nearly all world regions. These consisted of not being in the 35-49 year age group, residence in the lowest-income neighbourhoods, not being in a primary care patient enrolment model, a provider from the same region, and not having a female provider. For all women, the highest population-attributable risk was seen for not having a female provider, with values ranging from $16.8 \%$ [95\% Cl 14.6-19.1\%] among women from the Middle East and North Africa to $27.4 \%$ [95\% Cl 26.2-28.6\%] for women from East Asia and the Pacific.

Conclusions: To increase screening rates across immigrant groups, efforts should be made to ensure that women have access to a regular source of primary care, and ideally access to a female health professional. Efforts should also be made to increase the enrolment of immigrant women in new primary care patient enrolment models.
\end{abstract}

\section{Background}

Widespread screening using the Papanicolaou (Pap) test has been proven to dramatically reduce cervical cancer rates, and women who develop cervical cancer are most often women who have not been appropriately screened [1-5]. Therefore, in Ontario, Canada, evidence-based guidelines recommend that screening occur at least once every three years for all women with a history of vaginal sexual activity until 70 years of age[5]. However, the literature suggests that adherence to guidelines is not complete or equitable in our setting. Disparities in cervical cancer screening for foreign-born women have

\footnotetext{
* Correspondence: aisha.lofters@utoronto.ca

'Department of Family \& Community Medicine, University of Toronto,

Toronto, Canada

Full list of author information is available at the end of the article
}

long been documented in the Ontario and Canadian literature, using both self-report and administrative data [6-13]. This risk of non-screening is not equal across immigrant groups. In our previous work, although all immigrant groups had significantly lower screening rates than long-term residents of the province $[53.1 \%$ vs. 64.6\%), women from South Asia and from the Middle East and North Africa were the most vulnerable to lack of screening, and women from Western Europe and from Latin America and the Caribbean were the least vulnerable[14]. These findings were in the context of screening rates and knowledge about the Pap test being quite low in many South Asian and Middle Eastern countries. $[2,15,16]$.

The underlying mechanisms for screening disparities for immigrant women may lie in sociodemographic

\section{Biomed Central}


barriers, barriers rooted in the health care system, cultural or migration-related barriers or, most likely, some combination of the three [4,17-23]. As well, it is feasible that the relative importance of these barriers varies between ethnic groups. For example, the gender of the physician performing the Pap test or language barriers may be of more importance for women from one cultural or language group than from another. Therefore, the aims of this study were to: i) determine if the independent effects on cervical cancer screening of various factors reflective of sociodemographics, the health care system, and culture and migration were modified by region of origin for identified immigrant women in Ontario, and ii) to calculate populationattributable fractions for these factors for each region of origin.

\section{Methods Study Setting}

According to the 2006 Census, Ontario is Canada's largest province with a population of over 12 million people, over $28 \%$ of whom are foreign-born[24]. More than half of all Canada's immigrants settle in Ontario[25]. Asia is currently the main source continent, and India the number one source country, for newcomers to the province[25]. Nearly $75 \%$ of the province's population live in one of fifteen census metropolitan areas (CMAs) i.e. a geographic area with a total population of at least 100000 , of which 50000 or more live in an urban core $[26,27]$. For Ontario's foreign-born population, $94.0 \%$ live in a CMA[28]. Therefore, we limited the study setting to Ontario's CMAs. Ontario has a single, government-run, universal health insurance plan that pays for all medically necessary services, including cervical cancer screening.

\section{Data Sources}

Details on data sources have been previously published elsewhere[14].

\section{Study Cohort}

Our cohort consisted of all women in Ontario who were alive and eligible for health coverage from January 1 , 2006 to December 31, 2008, who ranged in age from 18 to 69 years for the entire three-year study period, whose most recent postal code was in a CMA, and who were identified immigrants based on the Landed Immigrant Data System (LIDS). A total of 524.997 women fit these initial inclusion criteria.

To ensure that we captured Pap tests performed for screening and not diagnostic purposes, we excluded women with an available history of gynaecological cancer in Ontario Cancer Registry records (1 427 women), or colposcopy in physicians' claims records (42 704 women). Women who have had a total hysterectomy are no longer screening-eligible, therefore we also excluded 26598 women with an available history of such in hospital discharge records. Ontario has newly instituted primary care patient enrolment models (PEMs), which include financial incentives for family physicians to perform cervical cancer screening on enrolled women aged 35-69 years. Because the tracking code Q140A can be claimed by Ontario physicians who participate in these models for any enrolled female patient aged 35-69 years who is ineligible for cervical cancer screening for any reason, we excluded 6008 women who had a Q140A code claimed at least once in available records. Due to overlap of reasons for exclusion, a total of 69133 women were excluded. Therefore, the final cohort consisted of 455864 women.

\section{Stratified Multivariate Analysis}

To classify women by region of origin, we accessed LIDS to determine country of birth for each woman. The countries were then grouped into regions based on a modification of the classification system used by the World Bank[29]. To determine if region of origin acted as an effect modifier, we conducted a stratified multivariate analysis by creating eight identical models, stratified by the eight world regions.

As our outcome is relatively common, odds ratios determined from logistic regression would not provide an accurate estimate of relative risks. Therefore, we used multivariate Poisson regression[30] to estimate adjusted relative risks. Models included variables in three categories: sociodemographic, health care-related and migration-related variables that may influence the likelihood of cervical cancer screening. For sociodemographic variables, we considered:

- age category (18-34 years, 35-49 years [referent], 5066 years) as there are financial incentives in PEMs for screening women 35 years and over and as women 50 years and over have previously been found to be underscreened[6,8,31]

- neighbourhood income quintile

- whether each woman lived in a small urban versus large urban setting based on her Rurality Index of Ontario score

- whether she had a university degree at the time of landing in Ontario. Health care-related variables included:

- whether each woman had at least one major prenatal visit during the study period, as Pap tests are expected aspects of these visit types and may be more acceptable during these visits

- whether she had seen a gynaecologist at least once during the study period

- whether she was rostered in a PEM 
- whether she was virtually rostered i.e. saw a family physician regularly who participates in a PEM despite the woman not being enrolled herself

- whether the woman had at least one female provider (either family physician or gynaecologist)

- whether she had at least one provider from the same region of the world (either family physician or gynaecologist) based on the physician's medical school

- the presence of co-morbidities in the two years prior to the index date based on the Johns Hopkins Adjusted Clinical Groups Case-Mix System, which. uses diagnostic information from administrative databases to describe and predict patients' use of health care resources. In this study, we used Resource Utilization Bands, which range from 0 (lowest expected health care costs) to 5 (highest expected health care costs), to categorize patients based on their expected use of health care resources, and Aggregated Diagnosis Groups, which range from 0 (no diagnosis group) to 32 (a maximum of 32 distinct diagnosis groups) to categorize the level of co-morbidity. This system has been validated for use in Canadian populations[32].

Migration-related variables included:

- immigrant class (economic [referent], family, refugee)

- English speaking ability at landing

- whether she had been in Canada less than 10 years

- age at landing.

SAS Version 9.1 (SAS Institute Inc, Cary, NC) was used to fit all models and determine adjusted relative risks.

Adjusted relative risks (ARRs) were then used to calculate population-attributable fractions (PAFs) for each variable using the following formula:

$$
\mathrm{PAF}=\mathrm{p}(\mathrm{ARR}-1) /(1+\mathrm{p}(\mathrm{ARR}-1))
$$

where $p$ was the proportion of the study population with the variable of interest.

\section{Outcome Definition}

To determine if each woman in the cohort had been appropriately screened for cervical cancer, we used a previously validated billing code-based algorithm consisting of all procedural codes that can be billed by the physician performing the Pap test and all laboratory codes that can be billed by the cytopathologist interpreting the Pap test. A woman was considered appropriately screened if at least one of the specified billing codes had been claimed for her in the threeyear study period. This algorithm had $99.5 \%$ sensitivity and $85.7 \%$ specificity when compared to a Pap test registry [6].

\section{Results}

Characteristics of the study population are summarized in Table 1. The largest immigrant groups were from East Asia and the Pacific (128 965 women) and from South Asia (88 107 women). The smallest group was from the USA, Australia and New Zealand (10 003 women). Women from Latin America and the Caribbean and from Sub-Saharan Africa were most likely to be living in the poorest neighbourhoods, and least likely to have a university degree. There was a mismatch between university-level education and income for South Asian women, with a high representation in lowincome neighbourhoods but a high level of educational attainment. Women from the USA, Australia and New Zealand had the highest educational attainment, and had the least amount of health care contact. South Asian women were the most likely to have at least one female provider and to have a provider from the same region of the world, and were most commonly rostered in PEMs. Eastern European and Central Asian women were the least likely to be able to speak English at landing, and women from Sub-Saharan Africa were the most likely to arrive as refugees.

A total of 213729 women (46.9\%) were not screened for cervical cancer during the three-year study period. Table 2 displays numbers and percentages of women who were not recently screened by region of origin for particular variables of interest. Women who had at least one female provider were the least likely to be unscreened, with the lowest number of unscreened women seen among Caribbean and Latin American women who had at least one female provider $(21.2 \%)$. The highest proportions of unscreened women were seen among those women who were neither in a PEM nor virtually rostered, with percentages consistently above $90 \%$. Among those women who were neither in a PEM nor virtually rostered, only $11.8 \%$ had any contact at all with the health care system during the study period (i.e. a physician office visit, hospitalization, emergency room visit, laboratory test, imaging procedure, or drug benefit claim). Of these women who had health care contact, $70.1 \%$ were still not recentlyly screened, ranging from $61.1 \%$ for women from the USA, Australia and New Zealand to $77.4 \%$ for Middle East and North African women (data not shown).

In our eight stratified models, there was little effect modification by region (Table 3 ). Certain variables were significantly associated with lack of screening across all or nearly all world regions. These consisted of being in either the youngest or oldest age groups and in the lowest income quintiles among the sociodemographic variables; and not being in a patient enrolment model, having a provider from the same region, and not having 
Table 1 Demographic characteristics of the 455864 identified immigrant women in the cohort who were aged 18-66 on January 1, 2006 by region of origin

\begin{tabular}{|c|c|c|c|c|c|c|c|c|c|}
\hline & $\begin{array}{c}\text { East } \\
\text { Asia } \\
\text { \&Pacific }\end{array}$ & $\begin{array}{c}\text { Eastern } \\
\text { Europe \& } \\
\text { Central Asia }\end{array}$ & $\begin{array}{c}\text { Latin } \\
\text { America \& } \\
\text { Caribbean }\end{array}$ & $\begin{array}{l}\text { Middle East } \\
\text { \& North } \\
\text { Africa }\end{array}$ & $\begin{array}{c}\text { South } \\
\text { Asia }\end{array}$ & $\begin{array}{c}\text { Sub- } \\
\text { Saharan } \\
\text { Africa }\end{array}$ & $\begin{array}{c}\text { USA, Australia } \\
\text { \& New } \\
\text { Zealand }\end{array}$ & $\begin{array}{c}\text { Western } \\
\text { Europe }\end{array}$ & $\begin{array}{c}\text { All } \\
\text { identified } \\
\text { Immigrants }\end{array}$ \\
\hline $\mathrm{n}$ & 128965 & 67845 & 70184 & 33649 & 88107 & 26125 & 10003 & 30167 & 455864 \\
\hline \multicolumn{10}{|c|}{ SOCIODEMOGRAPHIC FACTORS } \\
\hline Mean age (SD) & $\begin{array}{c}40.6 \\
(11.3)\end{array}$ & $39.5(11.8)$ & $38.8(11.8)$ & $37.2(12.1)$ & $\begin{array}{c}38.6 \\
(11.8)\end{array}$ & $\begin{array}{l}37.1 \\
(10.9)\end{array}$ & $39.7(11.8)$ & $\begin{array}{l}38.7 \\
(11.5)\end{array}$ & $39.2(11.7)$ \\
\hline \multicolumn{10}{|l|}{ Age category, No. (\%): } \\
\hline $18-34$ years & $\begin{array}{c}37321 \\
(28.9)\end{array}$ & $22413(33.0)$ & $25852(36.8)$ & $14333(42.6)$ & $\begin{array}{c}35565 \\
(40.4)\end{array}$ & $\begin{array}{c}10428 \\
(39.9)\end{array}$ & $3043(30.4)$ & $\begin{array}{c}10382 \\
(34.4)\end{array}$ & $\begin{array}{c}159581 \\
(35.0)\end{array}$ \\
\hline $35-49$ years & $\begin{array}{l}63857 \\
(49.5)\end{array}$ & $3137(45.9)$ & $30936(44.1)$ & $13743(40.8)$ & $\begin{array}{c}35904 \\
(40.8)\end{array}$ & $\begin{array}{l}12377 \\
(47.4)\end{array}$ & $4903(49.0)$ & $\begin{array}{l}14596 \\
(48.4)\end{array}$ & $\begin{array}{c}207836 \\
(45.6)\end{array}$ \\
\hline $50-66$ years & $\begin{array}{c}27787 \\
(21.6)\end{array}$ & $14295(21.1)$ & $13396(19.1)$ & $5573(16.6)$ & $\begin{array}{c}16638 \\
(18.9)\end{array}$ & $\begin{array}{l}3320 \\
(12.7)\end{array}$ & $2057(20.6)$ & $\begin{array}{l}5189 \\
(17.2)\end{array}$ & $88447(19.4)$ \\
\hline \multicolumn{10}{|l|}{ Income Quintile, No. (\%) } \\
\hline Q1 (lowest) & $\begin{array}{c}31755 \\
(24.6)\end{array}$ & $17591(25.9)$ & $25352(36.1)$ & $9045(26.9)$ & $\begin{array}{l}27878 \\
(31.6)\end{array}$ & $\begin{array}{c}11908 \\
(45.6)\end{array}$ & $1439(14.4)$ & $\begin{array}{l}5730 \\
(19.0)\end{array}$ & $\begin{array}{c}130867 \\
(28.7)\end{array}$ \\
\hline Q2 & $\begin{array}{l}32387 \\
(25.1)\end{array}$ & $13136(19.4)$ & $17696(25.2)$ & $6402(19.0)$ & $\begin{array}{l}23281 \\
(26.4)\end{array}$ & $\begin{array}{l}5042 \\
(19.3)\end{array}$ & $1591(15.9)$ & $\begin{array}{l}6788 \\
(22.5)\end{array}$ & $\begin{array}{c}106489 \\
(23.4)\end{array}$ \\
\hline Q3 & $\begin{array}{l}25862 \\
(20.1)\end{array}$ & $13403(19.8)$ & $13668(19.5)$ & $6511(19.4)$ & $\begin{array}{l}19038 \\
(21.6)\end{array}$ & $\begin{array}{l}3629 \\
(13.9)\end{array}$ & $1692(16.9)$ & $\begin{array}{l}5807 \\
(19.3)\end{array}$ & $89798(19.7)$ \\
\hline Q4 & $\begin{array}{l}22197 \\
(17.2)\end{array}$ & $14215(21.0)$ & $8518(12.1)$ & $6537(19.4)$ & $\begin{array}{c}11853 \\
(13.5)\end{array}$ & $\begin{array}{l}2985 \\
(11.4)\end{array}$ & $1948(19.5)$ & $\begin{array}{l}5800 \\
(19.2)\end{array}$ & $74218(16.3)$ \\
\hline Q5 (highest) & $\begin{array}{l}16556 \\
(12.8)\end{array}$ & $9434(13.9)$ & $4841(6.9)$ & $5105(15.2)$ & $\begin{array}{c}5964 \\
(6.8)\end{array}$ & $\begin{array}{c}2478 \\
(9.5)\end{array}$ & $3311(33.1)$ & $\begin{array}{l}5997 \\
(19.9)\end{array}$ & $53815(11.8)$ \\
\hline $\begin{array}{l}\text { No. (\%) with } \\
\text { university degree* }\end{array}$ & $\begin{array}{c}28369 \\
(22.0)\end{array}$ & $16729(24.7)$ & $4029(5.7)$ & $6832(20.3)$ & $\begin{array}{c}21923 \\
(24.9)\end{array}$ & $\begin{aligned} 2317 \\
(8.9)\end{aligned}$ & $3390(33.9)$ & $\begin{array}{c}2740 \\
(9.1)\end{array}$ & $86525(19.0)$ \\
\hline $\begin{array}{l}\text { No. (\%) living in large } \\
\text { urban area }\end{array}$ & $\begin{array}{c}124405 \\
(96.5)\end{array}$ & $62988(92.9)$ & $66975(95.5)$ & $31971(95.1)$ & $\begin{array}{c}85190 \\
(96.7) \\
\end{array}$ & $\begin{array}{c}25221 \\
(96.6)\end{array}$ & $8127(81.4)$ & $\begin{array}{c}26142 \\
(86.7)\end{array}$ & $\begin{array}{c}431805 \\
(94.8)\end{array}$ \\
\hline \multicolumn{10}{|c|}{ HEALTH CARE-RELATED FACTORS } \\
\hline \multicolumn{10}{|l|}{ No. (\%) in RUB category: } \\
\hline $0-1$ & $\begin{array}{l}34440 \\
(26.7)\end{array}$ & $13368(19.7)$ & $10967(15.6)$ & $8170(24.3)$ & $\begin{array}{l}13835 \\
(15.7)\end{array}$ & $\begin{array}{l}5591 \\
(21.4)\end{array}$ & $4592(45.9)$ & $\begin{array}{c}10138 \\
(33.6)\end{array}$ & $\begin{array}{c}101345 \\
(22.2)\end{array}$ \\
\hline 2 & $\begin{array}{l}19351 \\
(15.0)\end{array}$ & $10843(16.0)$ & $8439(12.0$ & $3946(11.7)$ & $\begin{array}{c}10419 \\
(11.8)\end{array}$ & $\begin{array}{l}3048 \\
(11.7)\end{array}$ & $1210(12.1)$ & $\begin{array}{l}3989 \\
(13.2)\end{array}$ & $61365(13.5)$ \\
\hline 3 & $\begin{array}{l}57936 \\
(44.9)\end{array}$ & $33743(49.7)$ & $36782(52.4)$ & $15170(45.1)$ & $\begin{array}{l}44120 \\
(50.1)\end{array}$ & $\begin{array}{c}11791 \\
(45.1)\end{array}$ & $3154(31.5)$ & $\begin{array}{l}12118 \\
(40.2)\end{array}$ & $\begin{array}{c}215149 \\
(47.2)\end{array}$ \\
\hline 4-5 & $\begin{array}{c}17238 \\
(13.4) \\
\end{array}$ & $9891(14.6)$ & $13996(19.9)$ & 6363 (18.9) & $\begin{array}{c}19733 \\
(22.4) \\
\end{array}$ & $\begin{array}{l}5695 \\
(21.8) \\
\end{array}$ & $1047(10.5)$ & $\begin{array}{l}3922 \\
(13.0) \\
\end{array}$ & $78005(17.1)$ \\
\hline \multicolumn{10}{|l|}{ No. (\%) in ADG category: } \\
\hline $0-5$ & $\begin{array}{l}83780 \\
(65.0)\end{array}$ & $42490(62.6)$ & $37137(52.9)$ & $19009(56.5)$ & $\begin{array}{l}44845 \\
(50.9)\end{array}$ & $\begin{array}{l}14426 \\
(55.2)\end{array}$ & $21046(69.8)$ & $\begin{array}{l}21046 \\
(69.8)\end{array}$ & $\begin{array}{c}270893 \\
(59.4)\end{array}$ \\
\hline $6-9$ & $\begin{array}{l}35926 \\
(27.9)\end{array}$ & $20473(30.2)$ & $24909(35.5)$ & $10527(31.3)$ & $\begin{array}{l}32035 \\
(36.4)\end{array}$ & $\begin{array}{l}8542 \\
(32.7)\end{array}$ & $7300(24.2)$ & $\begin{array}{l}7300 \\
(24.2)\end{array}$ & $\begin{array}{c}141848 \\
(31.1)\end{array}$ \\
\hline $10+$ & $\begin{array}{c}9259 \\
(7.2)\end{array}$ & $4882(7.2)$ & $8138(11.6)$ & $4113(12.2)$ & $\begin{array}{r}11227 \\
(12.7)\end{array}$ & $\begin{array}{l}3157 \\
(12.1)\end{array}$ & $1821(6.0)$ & $\begin{array}{r}1821 \\
(6.0) \\
\end{array}$ & $43123(9.5)$ \\
\hline $\begin{array}{l}\text { No. (\%) with prenatal } \\
\text { visit during study } \\
\text { period }\end{array}$ & $\begin{array}{c}5736 \\
(4.5)\end{array}$ & $3579(5.3)$ & $4610(6.6)$ & $1905(5.7)$ & $\begin{array}{c}7312 \\
(8.3)\end{array}$ & $\begin{array}{c}2000 \\
(7.7)\end{array}$ & $321(3.2)$ & $\begin{array}{l}1507 \\
(5.0)\end{array}$ & $26995(5.9)$ \\
\hline $\begin{array}{l}\text { No. (\%) in: Patient } \\
\text { enrolment model }\end{array}$ & $\begin{array}{c}80584 \\
(62.5)\end{array}$ & $41953(61.8)$ & $47620(67.9)$ & $21227(63.1)$ & $\begin{array}{l}63195 \\
(71.7)\end{array}$ & $\begin{array}{c}16682 \\
(63.9)\end{array}$ & $5101(51.0)$ & $\begin{array}{c}17707 \\
(58.7)\end{array}$ & $\begin{array}{c}294553 \\
(64.6)\end{array}$ \\
\hline Virtually rostered & $\begin{array}{l}23706 \\
(18.4)\end{array}$ & $17131(25.3)$ & $14979(21.3)$ & $6302(18.7)$ & $\begin{array}{c}15499 \\
(17.6)\end{array}$ & $\begin{array}{l}5374 \\
(20.6)\end{array}$ & $1123(11.2)$ & $\begin{array}{l}4482 \\
(14.9)\end{array}$ & $88732(19.5)$ \\
\hline Neither & $\begin{array}{c}24675 \\
(19.1)\end{array}$ & $8761(12.9)$ & $7585(10.8)$ & $6120(18.2)$ & $\begin{array}{l}9413 \\
(10.7)\end{array}$ & $\begin{array}{l}4069 \\
(15.6)\end{array}$ & $3779(37.8)$ & $\begin{array}{l}7978 \\
(26.5)\end{array}$ & $72579(15.9)$ \\
\hline $\begin{array}{l}\text { No. (\%) with female } \\
\text { provider }\end{array}$ & $\begin{array}{c}45676 \\
(35.4)\end{array}$ & 28917 (42.6) & 23234 (33.1) & 12555 (37.3) & $\begin{array}{l}42068 \\
(47.8)\end{array}$ & $\begin{array}{l}9679 \\
(37.1)\end{array}$ & $3188(31.9)$ & $\begin{array}{c}10866 \\
(36.0)\end{array}$ & $\begin{array}{c}176471 \\
(38.7)\end{array}$ \\
\hline
\end{tabular}


Table 1 Demographic characteristics of the 455864 identified immigrant women in the cohort who were aged 18-66 on January 1, 2006 by region of origin (Continued)

\begin{tabular}{|c|c|c|c|c|c|c|c|c|c|}
\hline $\begin{array}{l}\text { No. (\%) with provider } \\
\text { from same region }\end{array}$ & $\begin{array}{r}28081 \\
(21.8) \\
\end{array}$ & $19713(29.1)$ & $5397(7.7)$ & $8380(24.9)$ & $\begin{array}{c}34374 \\
(39.0) \\
\end{array}$ & $\begin{array}{c}1937 \\
(7.4) \\
\end{array}$ & $58(0.6)$ & $\begin{array}{r}2911 \\
(9.7) \\
\end{array}$ & $\begin{array}{c}100851 \\
(22.1) \\
\end{array}$ \\
\hline $\begin{array}{l}\text { No. (\%) with } \\
\text { gynaecologist }\end{array}$ & $\begin{array}{c}27739 \\
(21.5) \\
\end{array}$ & $20547(30.3)$ & $22028(31.4)$ & $8822(26.2)$ & $\begin{array}{c}23768 \\
(27.0) \\
\end{array}$ & $\begin{array}{l}7557 \\
(28.9) \\
\end{array}$ & $1472(14.7)$ & $\begin{array}{l}5985 \\
(19.8) \\
\end{array}$ & $\begin{array}{c}118075 \\
(25.9)\end{array}$ \\
\hline \multicolumn{10}{|c|}{ MIGRATION-RELATED FACTORS } \\
\hline $\begin{array}{l}\text { No. (\%) able to speak } \\
\text { English* }\end{array}$ & $\begin{array}{l}73105 \\
(56.7) \\
\end{array}$ & $24623(36.3)$ & $57012(81.2)$ & $17673(52.5)$ & $\begin{array}{c}43638 \\
(49.5) \\
\end{array}$ & $\begin{array}{c}18700 \\
(71.6) \\
\end{array}$ & $9675(96.7)$ & $\begin{array}{c}19901 \\
(66.0) \\
\end{array}$ & $\begin{array}{c}264848 \\
(58.1) \\
\end{array}$ \\
\hline $\begin{array}{l}\text { No. }(\%) \text { in Canada less } \\
\text { than } 10 \text { yrs }\end{array}$ & $\begin{array}{c}18931 \\
(14.7)\end{array}$ & $10919(16.1)$ & $6184(8.8)$ & $5578(16.6)$ & $\begin{array}{c}20179 \\
(22.9)\end{array}$ & $\begin{array}{l}3700 \\
(14.2)\end{array}$ & $1094(10.9)$ & $\begin{array}{l}1908 \\
(6.3)\end{array}$ & $68827(15.1)$ \\
\hline $\begin{array}{l}\text { Mean age at landing } \\
\text { (SD) }\end{array}$ & $\begin{array}{c}28.8 \\
(11.5)\end{array}$ & $27.8(12.0)$ & $25.5(12.0)$ & $25.8(12.4)$ & $\begin{array}{c}28.1 \\
(11.9)\end{array}$ & $\begin{array}{l}25.2 \\
(11.0)\end{array}$ & $26.3(12.1)$ & $\begin{array}{l}24.2 \\
(11.9)\end{array}$ & $27.2(11.9)$ \\
\hline \multicolumn{10}{|l|}{ Immigrant class, No. (\%) } \\
\hline Economic & $\begin{array}{l}74615 \\
(57.9)\end{array}$ & $26577(39.2)$ & $21684(30.9)$ & $16956(50.4)$ & $\begin{array}{c}30904 \\
(35.1)\end{array}$ & $\begin{array}{l}7669 \\
(29.4)\end{array}$ & $3508(35.1)$ & $\begin{array}{l}19438 \\
(64.4)\end{array}$ & $\begin{array}{c}201872 \\
(44.3)\end{array}$ \\
\hline Family & $\begin{array}{c}47632 \\
(36.7)\end{array}$ & $21099(31.1)$ & $41312(58.9)$ & $8688(25.8)$ & $\begin{array}{l}43243 \\
(49.1)\end{array}$ & $\begin{array}{l}8450 \\
(32.3)\end{array}$ & $6255(62.5)$ & $\begin{array}{l}9783 \\
(32.4)\end{array}$ & $\begin{array}{c}186444 \\
(40.9)\end{array}$ \\
\hline Refugee & $\begin{array}{c}5817 \\
(4.5)\end{array}$ & $19621(28.9)$ & $7010(10.0)$ & $7836(23.3)$ & $\begin{array}{l}13278 \\
(15.1)\end{array}$ & $\begin{array}{l}9834 \\
(37.6)\end{array}$ & $238(2.4)$ & $940(3.1)$ & $64618(14.2)$ \\
\hline
\end{tabular}

*Recorded on date of landing in Canada

RUB $=$ Resource Utilization Bands, which range from 0 (lowest expected health care costs) to 5 (highest expected health care costs), used to categorize patients based on their expected use of health care resources.

ADG = Aggregated Diagnosis Groups, which range from 0 (no diagnosis group) to 32 (32 distinct diagnosis groups) used to measure the level of co-morbidity.

a female provider among the health care-related variables. None of the migration-related variables were consistently significantly associated with lack of screening. Being unable to speak English at landing trended toward increased risk for most women, but equated to significantly decreased risk for East Asian and Pacific women. Immigrant class was only significant for Sub-Saharan African women and Western European women, with refugees being at higher risk of non-screening in these two groups. Post-hoc, we tested for an interaction between female provider and provider from the same region of the world, but it was not pervasively statistically significant so we did not include this interaction in the models.

We then determined PAFs for these variables (data not shown). For all women, the highest PAFs were seen for not having a female provider, with values ranging from 16.8\% [95\% CI 14.6-19.1\%] among women from the Middle East and North Africa to 27.4\% [95\% CI 26.2-28.6\%] for women from East Asia and the Pacific. The next highest PAFs varied by region of origin. Risk of non-screening could be attributed to being in the youngest age group for Latin American and Caribbean women (7.4\% [95\% CI 5.7-9.1\%]), Middle Eastern and North African women (7.5\% [95\% CI 5.1-9.9\%]), and South Asian women (7.7\% [95\% CI 6.4-9.1\%]). Being neither rostered nor virtually rostered in a primary care model was of especial importance for women from East Asia and the Pacific (5.9\% [95\% CI 5.3-6.5\%]), the USA, Australia and New Zealand (9.5\% [95\% CI 6.2-12.8\%]), and Western Europe (12.1\% [95\% CI 10.4-13.8\%]), as was not having a university degree for Eastern European and Central Asian women (6.9\% [95\% CI 4.7-9.1\%]), and being in the lowest income quintile for Sub-Saharan African women (8.9\% [95\% CI 5.7-12.1\%]).

\section{Discussion}

The cervical cancer screening rate of $53.1 \%$ that we have demonstrated for a three-year period for Ontario's immigrant women living in urban areas, all of whom were eligible for the provincial universal health care system, is substantially lower than would be expected with adherence to provincial guidelines[5], and substantially lower than the $64.6 \%$ we have previously found for long-term residents of Ontario living in urban areas during the same time period[14]. Sociodemographic and health care-related factors, namely living in the lowest-income neighbourhoods, not being in the 35-49 year age group, not being either rostered or virtually rostered in a patient enrolment model, and having either a male family doctor or a family doctor from the same region of the world were independently associated with lower rates of screening for immigrant women across most or all regions of origin, suggesting that these variables tend to negatively affect screening for immigrant women regardless of their culture or ethnicity. Even when limiting to women with at least one contact with the health care system during the study period, the prevalence of non-screening was still quite high for women without a family doctor, 
Table 2 Number (and percentage) of women without a Pap test in 2006-8 among the 455864 identified immigrant women in the cohort who were aged 18-66 on January 1, 2006 by region of origin

\begin{tabular}{|c|c|c|c|c|c|c|c|c|c|}
\hline & $\begin{array}{l}\text { East Asia } \\
\text { \& Pacific }\end{array}$ & $\begin{array}{c}\text { Eastern } \\
\text { Europe \& } \\
\text { Central Asia }\end{array}$ & $\begin{array}{l}\text { Latin } \\
\text { America \& } \\
\text { Caribbean } \\
\end{array}$ & $\begin{array}{l}\text { Middle East } \\
\text { \& North } \\
\text { Africa } \\
\end{array}$ & $\begin{array}{l}\text { South } \\
\text { Asia }\end{array}$ & $\begin{array}{c}\text { Sub- } \\
\text { Saharan } \\
\text { Africa }\end{array}$ & $\begin{array}{c}\text { USA, Australia } \\
\& \text { New } \\
\text { Zealand }\end{array}$ & $\begin{array}{c}\text { Western } \\
\text { Europe }\end{array}$ & $\begin{array}{c}\text { All } \\
\text { identified } \\
\text { immigrants }\end{array}$ \\
\hline \multicolumn{10}{|c|}{ SOCIODEMOGRAPHIC FA CTORS } \\
\hline \multicolumn{10}{|l|}{ Age category: } \\
\hline $18-34$ years & $\begin{array}{c}19513 \\
(52.3)\end{array}$ & $8640(38.6)$ & $9305(36.0)$ & $8082(56.4)$ & $\begin{array}{c}17828 \\
(50.1)\end{array}$ & $\begin{array}{l}5443 \\
(52.2)\end{array}$ & $1913(62.9)$ & $\begin{array}{l}4991 \\
(48.1)\end{array}$ & $75863(47.5)$ \\
\hline $35-49$ years & $\begin{array}{c}28916 \\
(45.3)\end{array}$ & $12327(39.6)^{\prime}$ & $10214(33.0)$ & $6358(46.3)$ & $\begin{array}{c}15689 \\
(43.7)\end{array}$ & $\begin{array}{l}5643 \\
(45.6)\end{array}$ & $2670(54.5)$ & $\begin{array}{l}7009 \\
(48.0)\end{array}$ & $89019(42.8)$ \\
\hline $50-66$ years & $\begin{array}{c}15053 \\
(54.2)\end{array}$ & $7456(52.2$ & $6412(47.9)$ & $3146(56.5)$ & $\begin{array}{c}10503 \\
(63.1)\end{array}$ & $\begin{array}{l}1768 \\
(53.3)\end{array}$ & $1344(65.3)$ & $\begin{array}{l}3057 \\
(58.9)\end{array}$ & $48847(55.2)$ \\
\hline \multicolumn{10}{|c|}{ Income Quintile } \\
\hline Q1 (lowest) & $\begin{array}{l}16980 \\
(53.5)\end{array}$ & $9102(51.7)$ & $10078(39.8)$ & $5375(59.4)$ & $\begin{array}{c}15358 \\
(55.1)\end{array}$ & $\begin{array}{l}6494 \\
(54.5)\end{array}$ & $976(67.8)$ & $\begin{array}{l}3330 \\
(58.1)\end{array}$ & $67778(51.8)$ \\
\hline Q2 & $\begin{array}{c}15677 \\
(48.4)\end{array}$ & $5827(44.4)$ & $6612(37.4)$ & $3472(54.2)$ & $\begin{array}{c}11534 \\
(49.5)\end{array}$ & $\begin{array}{l}2405 \\
(47.7)\end{array}$ & $985(61.9)$ & $\begin{array}{l}3522 \\
(51.9)\end{array}$ & $50126(47.1$ \\
\hline Q3 & $\begin{array}{c}11974 \\
(46.3)\end{array}$ & $5078(37.9)$ & $4519(33.1)$ & $3103(47.7)$ & $\begin{array}{l}8794 \\
(46.2)\end{array}$ & $\begin{array}{l}1658 \\
(45.7)\end{array}$ & $972(57.5)$ & $\begin{array}{l}2750 \\
(47.4)\end{array}$ & $38957(43.4)$ \\
\hline Q4 & $\begin{array}{c}10279 \\
(46.3)\end{array}$ & $4933(34.7)$ & $2839(33.3)$ & $3112(47.6)$ & $\begin{array}{l}5445 \\
(45.9)\end{array}$ & $\begin{array}{l}1225 \\
(41.0)\end{array}$ & $1063(54.6)$ & $\begin{array}{l}2580 \\
(44.5)\end{array}$ & $31565(42.5)$ \\
\hline Q5 (highest) & $\begin{array}{l}8443 \\
(51.0)\end{array}$ & 3441 (36.5) & $1832(37.8)$ & $2492(48.8)$ & $\begin{array}{l}2838 \\
(47.6)\end{array}$ & $\begin{array}{l}1017 \\
(41.0)\end{array}$ & $1913(57.8)$ & $\begin{array}{l}2845 \\
(47.4)\end{array}$ & $24893(46.3)$ \\
\hline
\end{tabular}

University degree:

\begin{tabular}{|c|c|c|c|c|c|c|c|c|c|}
\hline Yes & $\begin{array}{l}14141 \\
(49.9)\end{array}$ & $6605(39.5)$ & $1661(41.2)$ & $3495(51.2)$ & $\begin{array}{l}10426 \\
(45.1)\end{array}$ & $\begin{array}{l}1046 \\
(45.1)\end{array}$ & $1815(53.5)$ & $\begin{array}{l}1349 \\
(49.2)\end{array}$ & $40665(47.0)$ \\
\hline No & $\begin{array}{c}49341 \\
(49.1)\end{array}$ & $21818(42.7)$ & $24270(36.7)$ & $14091(52.6)$ & $\begin{array}{c}33594 \\
(50.8)\end{array}$ & $\begin{array}{c}11808 \\
(49.6)\end{array}$ & $4112(62.2)$ & $\begin{array}{l}13708 \\
(50.0)\end{array}$ & $\begin{array}{c}173064 \\
(46.9)\end{array}$ \\
\hline \multicolumn{10}{|c|}{ HEALTH CARE-RELATED FACTORS } \\
\hline $\begin{array}{l}\text { Patient enrolment } \\
\text { model }\end{array}$ & $\begin{array}{c}29196 \\
(36.2)\end{array}$ & $13403(32.0)$ & $13366(28.1)$ & $8545(40.3)$ & $\begin{array}{c}27200 \\
(43.0)\end{array}$ & $\begin{array}{l}6358 \\
(38.1)\end{array}$ & $1770(34.7)$ & $\begin{array}{l}5512 \\
(31.1)\end{array}$ & $\begin{array}{c}105538 \\
(35.8)\end{array}$ \\
\hline Virtually rostered & $\begin{array}{l}10262 \\
(43.3)\end{array}$ & $6803(39.7)$ & $5465(36.5)$ & $3072(48.8)$ & $\begin{array}{l}7707 \\
(49.7)\end{array}$ & $\begin{array}{l}2594 \\
(48.3)\end{array}$ & $450(40.1)$ & $\begin{array}{l}1760 \\
(39.3)\end{array}$ & $38177(43.0)$ \\
\hline Neither & $\begin{array}{c}24024 \\
(97.4)\end{array}$ & 8217 (93.8) & $7100(93.6)$ & $5969(97.5)$ & $\begin{array}{l}9113 \\
(96.8)\end{array}$ & $\begin{array}{l}3902 \\
(95.9)\end{array}$ & $3707(98.1)$ & $\begin{array}{l}7785 \\
(97.6)\end{array}$ & $70014(96.5)$ \\
\hline \multicolumn{10}{|l|}{ Female provider: } \\
\hline Yes & $\begin{array}{c}11434 \\
(25.0)\end{array}$ & $7359(25.5)$ & $4924(21.2)$ & $3971(31.6)$ & $\begin{array}{c}13947 \\
(33.2)\end{array}$ & $\begin{array}{l}2961 \\
(30.6)\end{array}$ & $873(27.4)$ & $\begin{array}{l}2653 \\
(24.4)\end{array}$ & $48220(27.3)$ \\
\hline No & $\begin{array}{l}52048 \\
(62.5)\end{array}$ & $21064(54.1)$ & $21007(44.7)$ & $13615(64.5)$ & $\begin{array}{c}30073 \\
(65.3)\end{array}$ & $\begin{array}{l}9893 \\
(60.2)\end{array}$ & $5054(74.2)$ & $\begin{array}{c}12404 \\
(64.3)\end{array}$ & $\begin{array}{c}165509 \\
(59.2)\end{array}$ \\
\hline \multicolumn{10}{|l|}{ region: } \\
\hline Yes & $\begin{array}{l}11279 \\
(40.2)\end{array}$ & $6474(32.8)$ & $1546(28.7)$ & $3653(43.6)$ & $\begin{array}{l}14760 \\
(42.9)\end{array}$ & $614(31.7)$ & $19(32.8)$ & $992(34.1)$ & $39337(39.0)$ \\
\hline No & $\begin{array}{l}52203 \\
(51.8)\end{array}$ & 21949 (45.6) & 24385 (37.6) & $13933(55.1)$ & $\begin{array}{l}29260 \\
(54.5)\end{array}$ & $\begin{array}{l}12240 \\
(50.6)\end{array}$ & $5908(59.4)$ & $\begin{array}{c}14065 \\
(51.6)\end{array}$ & $\begin{array}{c}174392 \\
(49.1)\end{array}$ \\
\hline \multicolumn{10}{|c|}{ MIGRATION-RELATED F ACTORS } \\
\hline \multicolumn{10}{|c|}{ Able to speak English: } \\
\hline Yes & $\begin{array}{l}36886 \\
(50.5)\end{array}$ & $10012(40.7)$ & 20479 (35.9) & $9165(51.9)$ & $\begin{array}{l}21656 \\
(49.6)\end{array}$ & $\begin{array}{l}8974 \\
(48.0)\end{array}$ & $5718(59.1)$ & $\begin{array}{l}9867 \\
(49.6)\end{array}$ & $\begin{array}{l}123036 \\
(46.5)\end{array}$ \\
\hline No & $\begin{array}{l}26596 \\
(47.6)\end{array}$ & $18411(42.6)$ & $5452(41.4)$ & $8421(52.7)$ & $\begin{array}{c}22364 \\
(50.3)\end{array}$ & $\begin{array}{l}3880 \\
(52.3)\end{array}$ & $209(63.7)$ & $\begin{array}{l}5190 \\
(50.6)\end{array}$ & $90693(47.5)$ \\
\hline
\end{tabular}




\begin{tabular}{|c|c|c|c|c|c|c|c|c|c|}
\hline \multicolumn{10}{|l|}{ In Canada: } \\
\hline less than 10 years & $\begin{array}{l}8055 \\
(42.6)\end{array}$ & $4381(40.1)$ & $2049(33.1)$ & $2840(50.9)$ & $\begin{array}{l}10272 \\
(50.9)\end{array}$ & $\begin{array}{l}1744 \\
(47.1)\end{array}$ & $541(49.5)$ & $851(44.6)$ & $30930(44.9)$ \\
\hline $10+$ years & $\begin{array}{l}55427 \\
(50.4)\end{array}$ & $24042(42.2)$ & $23882(37.3)$ & $14746(52.5)$ & $\begin{array}{l}33748 \\
(49.7)\end{array}$ & $\begin{array}{l}11110 \\
(49.5)\end{array}$ & $5386(60.5)$ & $\begin{array}{l}14206 \\
(50.3)\end{array}$ & $\begin{array}{l}182799 \\
(47.2)\end{array}$ \\
\hline \multicolumn{10}{|l|}{ Immigrant class: } \\
\hline Economic & $\begin{array}{c}39334 \\
(52.7)\end{array}$ & $10684(40.2)$ & $8599(39.7))$ & 9589 (56.6) & $\begin{array}{l}16810 \\
(54.4)\end{array}$ & $\begin{array}{l}3465 \\
(45.2)\end{array}$ & $2395(68.3)$ & $\begin{array}{l}9995 \\
(51.4)\end{array}$ & $\begin{array}{c}101173 \\
(50.1)\end{array}$ \\
\hline Family & $\begin{array}{l}21369 \\
(45.1)\end{array}$ & 9060 (42.9) & $14442(35.0)$ & $4150(47.8)$ & $\begin{array}{l}20514 \\
(47.4)\end{array}$ & $\begin{array}{l}3821 \\
(45.2)\end{array}$ & $3396(54.3)$ & $\begin{array}{l}4584 \\
(46.9)\end{array}$ & $81461(43.7)$ \\
\hline Refugee & $\begin{array}{l}2412 \\
(41.5)\end{array}$ & $8451(43.1)$ & $2822(40.3)$ & 3761 (48.0) & $\begin{array}{l}6357 \\
(47.9)\end{array}$ & $\begin{array}{l}5499 \\
(55.9)\end{array}$ & $135(56.7)$ & $475(50.5)$ & $29932(46.3)$ \\
\hline $\begin{array}{l}\text { TOTAL WITHOUT A } \\
\text { PAP TEST IN 2006-8 }\end{array}$ & $\begin{array}{c}63482 \\
(49.2)\end{array}$ & $28423(41.9)$ & $25931(36.9)$ & $17586(52.3)$ & $\begin{array}{c}44020 \\
(50.0)\end{array}$ & $\begin{array}{l}12854 \\
(49.2)\end{array}$ & $5927(59.2)$ & $\begin{array}{l}15057 \\
(49.9)\end{array}$ & $\begin{array}{c}213729 \\
(46.9)\end{array}$ \\
\hline
\end{tabular}

suggesting that complete lack of health care system contact did not explain this finding.

Our findings are similar to those of other studies that have shown that the gender and cultural origin of the family physician, and income and age of the patient, matter for cervical cancer screening. In another Canadian study, Decker et al. demonstrated that Canadian medical graduates and female physicians were more likely than international medical graduates and male physicians respectively to perform Pap tests[33]. In their literature review, Akers et al. noted that female doctors were consistently more likely to perform cervical screening and that having a doctor of the same ethnicity was associated with lower rates of screening[34]. Tu et al. showed that female physicians were more likely to screen for breast and cervical cancer among Chinese immigrants in both Seattle, USA and Vancouver, Canada[35], and in qualitative studies, immigrant women consistently report that having a female perform the Pap test would increase their comfort level[36-40]. Low-income women have frequently been highlighted as vulnerable to under-screening, both among foreign-born women and among the general population $[2,6-8,23,33,41,42]$. Although many international studies have shown that older age is associated with lower rates of cervical cancer screening among both immigrants and the general population[2,3,8,40,43-49], only a few studies have highlighted women in the youngest age group as vulnerable to under-screening and most of these have focussed on women younger than 25 years [23,49-51]. It must be noted that the benefits of screening for women under 25 years may be limited[52].

We also used PAFs to determine the screening barriers of most importance for each cultural group, and found that access to a female provider had the highest attributable risk across regions of origin. Other characteristics which decision makers could focus on were also highlighted with some differences across regions. These findings can be used for screening interventions that are targeted at particular ethnic groups. For example, researchers and policymakers aiming to increase screening among Sub-Saharan African women may wish to focus their efforts on women living in the poorest neighbourhoods.

\section{Strengths and Limitations}

This study has several strengths. It is a large, population-based study with broad inclusion criteria that distinguishes immigrant women from all major geographic regions of the world. It uses a previously validated outcome measure[6] instead of self-report to document cervical cancer screening. Self-report is known to systematically overestimate screening attendance[53,54]., It also relies on objective data instead of self-reported data for immigration status and region of origin. The effects of sociodemographics, health care-related factors and migration-related factors on screening for immigrant women from all regions of the world were considered. As well, this is the only study that we are aware of that has examined region of origin as a potential source of effect modification and calculated region-specific population-attributable fractions in order to determine barriers to Pap test use of the most importance for each cultural group.

This study also has several limitations. First, not all potentially relevant information, such as religion, is available from administrative data. Second, some data were only available for women at the time of landing, such as education attainment and language ability. These may have changed for many women by the beginning of the study period. Third, identified immigrants were classified based on their country of birth, which 
Table 3 Adjusted relative risks [with $95 \%$ confidence intervals] for risk of non-screening for the 455864 identified immigrant women in the cohort who were aged 18-66 on January 1, 2006 by region of origin

\begin{tabular}{|c|c|c|c|c|c|c|c|c|c|}
\hline & $\begin{array}{l}\text { East Asia \& } \\
\text { Pacific }\end{array}$ & $\begin{array}{l}\text { Eastern Europe \& } \\
\text { Central Asia }\end{array}$ & $\begin{array}{l}\text { Latin America \& } \\
\text { Caribbean }\end{array}$ & $\begin{array}{l}\text { Middle East \& } \\
\text { North Africa }\end{array}$ & South Asia & $\begin{array}{l}\text { Sub-Saharan } \\
\text { Africa }\end{array}$ & $\begin{array}{l}\text { USA, Australia \& } \\
\text { New Zealand }\end{array}$ & $\begin{array}{l}\text { Western } \\
\text { Europe }\end{array}$ & $\begin{array}{l}\text { All identified } \\
\text { immigrants }\end{array}$ \\
\hline \multicolumn{10}{|c|}{ SOCIODEMOGRAPHIC FACTORS } \\
\hline \multicolumn{10}{|l|}{ Age category: } \\
\hline $18-34$ years & $1.20[1.16-1.23]$ & $1.16[1.11-1.21]$ & $1.22[1.17-1.27]$ & $1.19[1.13-1.26]$ & $1.21[1.17-1.25]$ & $1.16[1.10-1.24]$ & $1.13[1.03-1.25]$ & $1.19[1.12-1.27]$ & $1.24[1.22-1.26]$ \\
\hline $35-49$ years & 1.0 & 1.0 & 1.0 & 1.0 & 1.0 & 1.0 & 1.0 & 1.0 & 1.0 \\
\hline $50-66$ years & $1.20[1.17-1.24]$ & $1.08[1.04-1.13]$ & $1.24[1.19-1.30]$ & $1.16[1.10-1.23]$ & $1.30[1.25-1.35]$ & $1.09[1.02-1.17]$ & $1.06[0.97-1.16]$ & $1.10[1.04-1.17]$ & 1.15 [1.13-1.17] \\
\hline Q1 (lowest) & 1.10 [1.08-1.13] & $1.15[1.11-1.20]$ & $1.10[1.05-1.16]$ & 1.14 [1.08-1.19] & $1.12[1.08-1.17]$ & 1.21 [1.13-1.30] & 1.09 [1.00-1.18] & $1.12[1.06-1.18]$ & $1.14[1.12-1.15]$ \\
\hline Q2 & $1.03[1.01-1.06]$ & $1.09[1.05-1.14]$ & $1.06[1.00-1.11]$ & $1.09[1.03-1.15]$ & $1.07[1.03-1.11]$ & $1.12[1.04-1.20]$ & $1.06[0.98-1.15]$ & $1.09[1.03-1.14]$ & 1.07 [1.06-1.09] \\
\hline Q3 & $1.01[0.98-1.04]$ & $1.03[0.99-1.08]$ & $0.97[0.92-1.03]$ & $1.01[0.96-1.07]$ & $1.02[0.98-1.07]$ & $1.12[1.03-1.21]$ & $1.03[0.95-1.11]$ & $1.06[1.01-1.12]$ & $1.03[1.01-1.04]$ \\
\hline Q4 & $0.99[0.96-1.02]$ & $1.00[0.95-1.04]$ & $0.97[0.91-1.03]$ & $1.01[0.96-1.07]$ & $1.00[0.96-1.05]$ & $1.01[0.93-1.10]$ & $0.99[0.91-1.06]$ & $1.00[0.95-1.06]$ & $1.0[0.98-1.01]$ \\
\hline Q5 (highest) & 1.0 & 1.0 & 1.0 & 1.0 & 1.0 & 1.0 & 1.0 & 1.0 & 1.0 \\
\hline \multicolumn{10}{|l|}{ University degree: } \\
\hline No & $0.98[0.96-1.00]$ & $1.10[1.07-1.13]$ & $1.05[0.99-1.10]$ & $1.01[0.97-1.05]$ & $1.06[1.03-1.08]$ & $1.03[0.96-1.10]$ & $1.06[1.00-1.13]$ & $1.04[0.98-1.11]$ & 1.01 [1.00-1.02] \\
\hline Yes & 1.0 & 1.0 & 1.0 & 1.0 & 1.0 & 1.0 & 1.0 & 1.0 & 1.0 \\
\hline \multicolumn{10}{|c|}{ HEALTH CARE-RELATED FACTORS } \\
\hline No family doctor & $1.33[1.30-1.36]$ & $1.47[1.41-1.52]$ & $1.56[1.49-1.62]$ & $1.31[1.24-1.37]$ & $1.21[1.17-1.26]$ & 1.31 [1.24-1.39] & $1.28[1.17-1.39]$ & $1.52[1.44-1.60]$ & 1.39 [1.37-1.41] \\
\hline Virtually rostered & $1.19[1.16-1.21]$ & $1.23[1.19-1.27]$ & $1.28[1.24-1.32]$ & 1.18 [1.13-1.23] & $1.13[1.10-1.16]$ & $1.21[1.15-1.26]$ & $1.17[1.05-1.29]$ & $1.26[1.20-1.33]$ & $1.18[1.17-1.20]$ \\
\hline $\begin{array}{l}\text { Patient enrolment } \\
\text { model }\end{array}$ & 1.0 & 1.0 & 1.0 & 1.0 & 1.0 & 1.0 & 1.0 & 1.0 & 1.0 \\
\hline \multicolumn{10}{|l|}{ Female provider: } \\
\hline No & $1.58[1.55-1.62]$ & $1.44[1.40-1.49]$ & $1.40[1.35-1.45]$ & $1.32[1.27-1.38]$ & $1.44[1.41-1.47]$ & $1.33[1.27-1.39]$ & $1.43[1.31-1.55]$ & $1.44[1.38-1.52]$ & $1.43[1.41-1.45]$ \\
\hline Yes & 1.0 & 1.0 & 1.0 & 1.0 & 1.0 & 1.0 & 1.0 & 1.0 & 1.0 \\
\hline \multicolumn{10}{|l|}{$\begin{array}{l}\text { Provider from } \\
\text { same region: }\end{array}$} \\
\hline Yes & $1.06[1.04-1.09]$ & $1.08[1.05-1.12]$ & $1.08[1.02-1.13]$ & $1.13[1.08-1.17]$ & $1.09[1.07-1.11]$ & $0.94[0.86-1.02]$ & $0.98[0.62-1.53]$ & $1.14[1.07-1.22]$ & $1.15[1.13-1.16]$ \\
\hline No & 1.0 & 1.0 & 1.0 & 1.0 & 1.0 & 1.0 & 1.0 & 1.0 & 1.0 \\
\hline \multicolumn{10}{|c|}{ MIGRATION-RELATED FACTORS } \\
\hline \multicolumn{10}{|c|}{ Able to speak English } \\
\hline No & $0.98[0.96-0.99]$ & $1.01[0.98-1.04]$ & $1.04[1.01-1.08]$ & $1.04[1.00-1.07]$ & $1.09[1.06-1.11]$ & $1.06[1.02-1.10]$ & $1.05[0.90-1.22]$ & $1.03[1.00-1.07]$ & $1.04[1.03-1.05]$ \\
\hline Yes & 1.0 & 1.0 & 1.0 & 1.0 & 1.0 & 1.0 & 1.0 & 1.0 & 1.0 \\
\hline \multicolumn{10}{|l|}{ In Canada: } \\
\hline less than 10 years & 1.00 [0.98-1.03] & 1.01 [0.98-1.05] & $0.97[0.92-1.02]$ & $1.06[1.02-1.11]$ & 1.09 [1.06-1.11] & $1.05[0.99-1.11]$ & 1.10 [1.00-1.22] & $1.05[0.97-1.13]$ & 1.05 [1.04-1.07] \\
\hline $10+$ years & 1.0 & 1.0 & 1.0 & 1.0 & 1.0 & 1.0 & 1.0 & 1.0 & 1.0 \\
\hline \multicolumn{10}{|l|}{ Immigrant class: } \\
\hline Family & $1.00[0.99-1.02]$ & 1.01 [0.98-1.04] & $0.98[0.95-1.01]$ & $0.96[0.93-1.00]$ & 0.98 [0.95-1.00] & 1.05 [1.00-1.10] & $0.97[0.91-1.02]$ & 0.98 [0.95-1.02] & 0.99 [0.98-1.00] \\
\hline Refugee & $0.96[0.92-1.00]$ & 1.00 [0.97-1.03] & 1.03 [0.99-1.08] & 1.00 [0.96-1.04] & 1.02 [0.99-1.05] & 1.20 [1.15-1.26] & 1.07 [0.89-1.29] & 1.08 [0.98-1.18] & 1.06 [1.04-1.07] \\
\hline Economic & 1.0 & 1.0 & 1.0 & 1.0 & 1.0 & 1.0 & 1.0 & 1.0 & 1.0 \\
\hline
\end{tabular}

Relative risks adjusted for all variables listed in the table. 
may not always reflect their ethnic and cultural origins. For example, 238 women born in the U.S. were in the refugee class, most likely reflective of women of other ethnic origins whose families lived for a time in the U.S. before settling in Canada. Fourth, although we excluded women where there was any evidence of a hysterectomy in available data, we could not identify out-of-province hysterectomies, which may be relatively common among the immigrant women in the cohort. However, we also excluded women who had a Q140 code billed, which allows physicians in primary care enrolment models to flag those patients whom they have deemed ineligible for screening. All identified immigrants had been in Ontario for over five years by the first day of the study period, and on average, had been in the province for over 10 years, decreasing the likelihood of out-of-province hysterectomies. Finally, our results may not be generalizable to other settings, either inside or outside Canada, as other settings may have different immigrant demographic profiles.

\section{Conclusions}

Our findings suggest that several interventions may be beneficial for improving cervical cancer screening rates among immigrant women in Ontario. First, efforts need to be made to ensure that immigrant women get connected with the health care system after arrival and find a regular source of primary care. Settlement agencies may be able to play a substantial role toward this goal. Moving from Ontario's current system of opportunistic screening to one of centrally organized screening with periodic invitations may also be of benefit for increasing screening rates. Although it is neither feasible nor desirable for every immigrant woman to see a female provider, efforts should also be made to increase the enrolment of immigrant women in primary care patient enrolment models. Importantly, some primary care models may also make it feasible for male physicians to have female health professionals, such as trained nurses, physician assistants or nurse practitioners, available to provide cervical cancer screening, which may increase immigrant women's comfort with having the procedure performed. As well, targeted physician education campaigns for physicians trained abroad may be beneficial for improving screening rates. Future work should examine the reasons for lower screening rates when there is ethnic congruence between a physician and patient. Targeted patient education campaigns and interventions for all immigrant women will likely also be of utmost importance, with a particular focus on younger and older women, and on women of low income.

\section{Acknowledgements}

Data were provided by Citizenship and Immigration Canada and the Institute for Clinical Evaluative Sciences (ICES). Dr. Aisha Lofters is supported by a Canadian Institutes For Health Research (CIHR) Doctoral Award and a Primary Health Care System Research Fellowship. The Canadian Population Health Initiative of the Canadian Institute for Health Information, Citizenship and Immigration Canada and the Public Health Agency of Canada (PHAC) sponsored the data linkage for this study. This study was also supported by ICES and the Centre for Research on Inner City Health (CRICH), which are funded by an annual grant from the Ontario Ministry of Health and LongTerm Care (MOHLTC). The opinions, results and conclusions reported in this paper are those of the authors and are independent from the funding sources. No endorsement by ICES, CRICH or the Ontario MOHLTC is intended or should be inferred.

\section{Author details}

${ }^{1}$ Department of Family \& Community Medicine, University of Toronto, Toronto, Canada. ${ }^{2}$ Department of Family \& Community Medicine, St. Michael's Hospital, Toronto, Canada. ${ }^{3}$ Centre for Research on Inner City Health, The Keenan Research Centre in the Li Ka Shing Knowledge Institute of St. Michael's Hospital, Toronto, Canada. ${ }^{4}$ Primary Health Care System Research Fellow, London, Canada. ${ }^{5}$ Institute for Clinical Evaluative Sciences, Toronto, Canada. ${ }^{6}$ Dalla Lana School of Public Health, University of Toronto, Toronto, Canada. ${ }^{7}$ Department of Medicine, University of Toronto, Toronto, Canada.

\section{Authors' contributions}

$\mathrm{AL}$ took primary responsibility for the design of the study, the acquisition, analysis and interpretation of data, and drafting and revising the article. RM provided advice and direction for the study design and contributed substantially to data analysis and interpretation. SH provided advice and direction for the study design and contributed substantially to data interpretation. RG took primary responsibility for the conception of the study. He provided advice and direction for the study design, and data acquisition, analysis and interpretation. All authors revised the article critically for important intellectual content and gave final approval of the version to be published.

\section{Competing interests}

The authors declare that they have no competing interests.

Received: 22 December 2010 Accepted: 27 May 2011

Published: 27 May 2011

\section{References}

1. Sankaranarayanan R, Budukh AM, Rajkumar R: Effective screening programmes for cervical cancer in low- and middle-income developing countries. Bull World Health Organ 2001, 79(10):954-62.

2. Gakidou E: Coverage of cervical cancer screening in 57 countries: low average levels and large inequalities. PLoS medicine 2008, 5(6):132

3. Owusu GA, Eve SB, Cready CM, Koelln K, Trevino F, Urrutia-Rojas X, Baumer J: Race and ethnic disparities in cervical cancer screening in a safety-net system. Matern Child Health J 2005, 9(3):285-95.

4. CancerCareOntario: Ontario Cervical Screening Program, Program Report 2001-2005. Toronto, ON

5. McLachlin CM, Mai V, Murphy J, Fung Kee Fung M, Chambers A: Cervical screening: a clinical practice guideline. In Ontario Cervical Screening Program GCDSG. Edited by: Toronto ON. Cancer Care Ontario; 2005:

6. Lofters AK, Moineddin R, Hwang SW, Glazier RH: Low rates of cervical cancer screening among urban immigrants: a population-based study in Ontario, Canada. Med Care 2010, 48(7):611-8.

7. Lofters A, Glazier RH, Agha MM, Creatore Ml, Moineddin R: Inadequacy of cervical cancer screening among urban recent immigrants: a population-based study of physician and laboratory claims in Toronto, Canada. Prev Med 2007, 44(6):536-42.

8. Maxwell CJ, Bancej CM, Snider J, Vik SA: Factors important in promoting cervical cancer screening among Canadian women: findings from the 1996-97 National Population Health Survey (NPHS). Can J Public Health 2001, 92(2):127-33. 
9. Xiong H, Murphy M, Mathews M, Gadag V, Wang PP: Cervical cancer screening among Asian Canadian immigrant and nonimmigrant women. Am J Health Behav 2010, 34(2):131-43

10. Woltman KJ, Newbold KB: Immigrant women and cervical cancer screening uptake: a multilevel analysis. Can J Public Health 2007, 98(6):470-5.

11. Brotto LA, Chou AY, Singh T, Woo JS: Reproductive health practices among Indian, Indo-Canadian, Canadian East Asian, and Euro-Canadian women: the role of acculturation. Journal of Obstetrics \& Gynaecology Canada: JOGC 2008, 30(3):229-38.

12. McDonald JT, Kennedy S: Cervical cancer screening by immigrant and minority women in Canada. Journal of immigrant and minority health 2007, 9(4):323.

13. Amankwah $\mathrm{E}$, Ngwakongnwi $\mathrm{E}$, Quan $\mathrm{H}$ : Why many visible minority women in Canada do not participate in cervical cancer screening. Ethn Health 2009, 14(4):337-49.

14. Lofters AK, Hwang SW, Moineddin R, Glazier RH: Cervical cancer screening among urban immigrants by region of origin: A population-based cohort study. Prev Med 2010.

15. Barghouti FF, Takruri AH, Froelicher ES: Awareness and behavior about Pap smear testing in family medicine practice. Saudi Med J 2008, 29(7):1036-40

16. Al Sairafi M, Mohamed FA: Knowledge, attitudes, and practice related to cervical cancer screening among Kuwaiti women. Med Princ Pract 2009, 18(1):35-42.

17. Wang JH, Sheppard VB, Schwartz MD, Liang W, Mandelblatt JS: Disparities in cervical cancer screening between Asian American and Non-Hispanic white women. Cancer Epidemiol Biomarkers Prev 2008 17(8):1968-73.

18. Johnson CE, Mues KE, Mayne SL, Kiblawi AN: Cervical cancer screening among immigrants and ethnic minorities: a systematic review using the Health Belief Model. J Low Genit Tract Dis 2008, 12(3):232-41.

19. Schleicher E: Immigrant Women and Cervical Cancer Prevention in the United States. Baltimore: Women's and Children's Health Policy Center Johns Hopkins Bloomberg School of Public Health; 2007.

20. Webb R, Richardson J, Pickles A: A population-based study of primary care predictors of non-attendance for cervical screening. Journal of medical screening 2004, 11(3):135-40.

21. Hislop TG, Inrig KM, Bajdik CD, Deschamps M, Tu SP, Taylor VM: Health care services and Pap testing behavior for Chinese women in British Columbia. J Immigr Health 2003, 5(4):143-52

22. Hislop TG, Teh C, Lai A, Ralston JD, Shu J, Taylor VM: Pap screening and knowledge of risk factors for cervical cancer in Chinese women in British Columbia, Canada. Ethn Health 2004, 9(3):267-81.

23. Krzyzanowska M, Barbera L, Elit L, Kwon J, Lofters A, Saskin R, Yeritsan N, Bierman A: In Cancer Project for an Ontario Women's Health Evidence-Based Report Toronto Edited by: AS B 2009.

24. Census: Toronto: CHASS; 2006 [http://dc2.chass.utoronto.ca.myaccess.library. utoronto.ca/census/2006/, [updated November 13 2008; cited 2009].

25. MinistryofFinance: Census Highlights: Fact Sheet 7, Immigration and Citizenship. 2006 [http://www.fin.gov.on.ca/en/economy/demographics/ census/cenhi06-7.pdf], [November 7, 2009].

26. MinistryofFinance: Census Highlights: Factsheet 1. Population Counts: Canada, Ontario and Regions.: Queen's Printer for Ontario; 2006 [http:// www.fin.gov.on.ca/en/economy/demographics/census/cenhi06-1.html], [updated October 7, 2009].

27. Census: Highlights: Factsheet 1: Population Growth in Ontario's CMAs and the GTA. Ministry of Finance; 2007, [document] 2001 [http://www.fin gov.on.ca/english/economy/demographics/census/cenhi1.html].

28. 2006 Census Profile. Toronto: Canadian Census Analyser @ CHASS; [http:// dc2.chass.utoronto.ca.myaccess.library.utoronto.ca/census/2006/], [updated November 13, 2008]

29. Country Classifications, The World Bank: The World Bank Group. 2010 [http://data.worldbank.org/about/country-classifications].

30. McNutt $L A$, Wu $C$, Xue $X$, Hafner JP: Estimating the relative risk in cohort studies and clinical trials of common outcomes. Am J Epidemiol 2003, 157(10):940-3.

31. Finkelstein M: Preventive screening. What factors influence testing? Canadian family physician 2002, 48:1494-501.
32. Reid RJ, MacWilliam L, Verhulst L, Roos N, Atkinson M: Performance of the ACG case-mix system in two Canadian provinces. Med Care 2001, 39(1):86-99.

33. Decker K, Demers A, Chateau D, Must G, Nugent Z, Lotocki R, Harrison M: Papanicolaou test utilization and frequency of screening opportunities among women diagnosed with cervical cancer. Open Medicine 2009, 3(3):140-7

34. Akers AY, Newmann SJ, Smith JS: Factors underlying disparities in cervical cancer incidence, screening, and treatment in the United States. Current problems in cancer 2007, 31(3):157-81.

35. Tu SP, Jackson SL, Yasui Y, Deschamps M, Hislop TG, Taylor VM: Cancer preventive screening: a cross-border comparison of United States and Canadian Chinese women. Prev Med 2005, 41(1):36-46.

36. Black ME, Frisina A, Hack T, Carpio B: Improving early detection of breast and cervical cancer in Chinese and Vietnamese immigrant women. Oncology Nursing ForumOnline 2006, 33(5):873-6.

37. Donnelly $\Pi$ : The health-care practices of Vietnamese-Canadian women: cultural influences on breast and cervical cancer screening. Canadian Journal of Nursing Research 2006, 38(1):82-101.

38. Lesjak M, Hua M, Ward J: Cervical screening among immigrant Vietnamese women seen in general practice: current rates, predictors and potential recruitment strategies. Aust N Z J Public Health 1999 23(2):168-73

39. Donnelly $\Pi$ : Challenges in providing breast and cervical cancer screening services to Vietnamese Canadian women: the healthcare providers' perspective. Nursing inquiry 2008, 15(2):158-68.

40. Oelke ND, Vollman AR: "Inside and outside": Sikh women's perspectives on cervical cancer screening. Canadian Journal of Nursing Research 2007, 39(1):174-89.

41. Coughlin SS, King J, Richards TB, Ekwueme DU: Cervical cancer screening among women in metropolitan areas of the United States by individuallevel and area-based measures of socioeconomic status, 2000 to 2002 Cancer Epidemiology, Biomarkers \& Prevention 2006, 15(11):2154-9.

42. Lee EE, Fogg L, Menon U: Knowledge and beliefs related to cervical cancer and screening among Korean American women. West J Nurs Res 2008, 30(8):960-74

43. Seidel D, Becker N, Rohrmann S, Nimptsch K, Linseisen J: Sociodemographic characteristics of participation in the opportunistic German cervical cancer screening programme: results from the EPICHeidelberg cohort. J Cancer Res Clin Oncol 2009, 135(4):533-41.

44. Johnston GM, Boyd CJ, Maclsaac MA: Community-based cultural predictors of Pap smear screening in Nova Scotia. Can J Public Health 2004, 95(2):95-8.

45. Blackwell DL, Martinez ME, Gentleman JF: Women's compliance with public health guidelines for mammograms and pap tests in Canada and the United States: an analysis of data from the Joint Canada/United States Survey Of Health. Womens Health Issues 2008, 18(2):85-99.

46. Simoes EJ, Newschaffer CJ, Hagdrup N, Ali-Abarghoui F, Tao X, Mack N, Brownson RC: Predictors of compliance with recommended cervical cancer screening schedule: a population-based study. J Community Health 1999, 24(2):115-30.

47. Mishra SI, Luce-Aoelua PH, Hubbell FA: Predictors of papanicolaou smear use among american samoan women. J Gen Intern Med 2001, 16(5):320-4.

48. Cabeza E, Esteva M, Pujol A, Thomas V Sanchez-Contador C. Social disparities in breast and cervical cancer preventive practices. Eur J Cancer Prev 2007, 16(4):372-9.

49. Wang PD, Lin RS: Sociodemographic factors of Pap smear screening in Taiwan. Public Health 1996, 110(2):123-7.

50. Rodvall Y, Kemetli L, Tishelman C, Tornberg S: Factors related to participation in a cervical cancer screening programme in urban Sweden. Eur J Cancer Prev 2005, 14(5):459-66.

51. Matuk LC: Pap smear screening practices in newcomer women. Womens Health Issues 1996, 6(2):82-8.

52. Sasieni $P$, Castanon A, Cuzick J: Effectiveness of cervical screening with age: population based case-control study of prospectively recorded data. BMJ 2009, 339:b2968.

53. Arbyn M, Simoens C, Van Oyen H, Foidart JM, Goffin F, Simon P, Fabri V Analysis of 13 million individual patient records pertaining to Pap smears, colposcopies, biopsies and surgery on the uterine cervix (Belgium, 1996-2000). Prev Med 2009, 48(5):438-43. 
54. Montano DE, Phillips WR: Cancer screening by primary care physicians: a comparison of rates obtained from physician self-report, patient survey, and chart audit. Am J Public Health 1995, 85(6):795-800.

\section{Pre-publication history}

The pre-publication history for this paper can be accessed here: http://www.biomedcentral.com/1472-6874/11/20/prepub

doi:10.1186/1472-6874-11-20

Cite this article as: Lofters et al.: Predictors of low cervical cancer

screening among immigrant women in Ontario, Canada. BMC Women's Health 2011 11:20

Submit your next manuscript to BioMed Central and take full advantage of:

- Convenient online submission

- Thorough peer review

- No space constraints or color figure charges

- Immediate publication on acceptance

- Inclusion in PubMed, CAS, Scopus and Google Scholar

- Research which is freely available for redistribution 